\title{
Puerpério remoto: seguimento do primeiro ano pós-parto pela atenção primária de saúde
}

\author{
Puerperium remote: first year period postpartum for primary health warning
}

Puerperio remoto: seguimiento del primer año posparto por atención primaria de salud

Áurea Fabrícia Amâncio Quirino Silva ${ }^{1 *}$, Alexandrina Aparecida Maciel Cardelli², Rosângela Aparecida Pimenta Ferrari², Fabiana Fontana Medeiros ${ }^{2}$, Izabel Dayana de Lemos Santos ${ }^{2}$, Franciane Maria da Silva Curan², Juliana Vicente de Oliveira Franchi².

\section{RESUMO}

Objetivo: Identificar as possíveis lacunas no seguimento puerperal pelo serviço de saúde e a procura por cuidado pela mulher no primeiro ano pós-parto. Métodos: Coorte prospectiva, com 310 mulheres, em um município do Paraná, entre julho de 2013 a março de 2015. Realizou-se análise estatísca dos dados por meio do Teste Qui-quadrado e do Coeficiente de Spearman. Resultados: Encontrou-se significância estatística entre ter adoecido ( $p \leq 0,024)$; dificuldade para urinar $(p \leq 0,011)$; autopercepção de doença depressiva $(p \leq$ $0,007)$; realização de ações para melhorar os sinais e sintomas de depressão $(p \leq 0,007)$; citologia oncótica $(p \leq 0,000)$; procura por cuidado até 42 dias após o parto $(p \leq 0,031)$ e entre 43 dias e um ano após o parto $(p \leq 0,012)$; satisfação com atendimento multiprofissional ( $p \leq 0,001)$, médico $(p \leq 0,000)$, de enfermagem ( $p$ $\leq 0,000$ ); e as variáveis. O Coeficiente de Spearman evidenciou correlação muito forte entre realização de revisão puerperal precoce domiciliar (RPPD) e agendamento da revisão puerperal tardia na UBS na RPP $(r=$ 0,953). Conclusão: A lacuna identificada no puerpério foi de abandono assistencial e a procura por cuidado evidenciou a tentativa das mães de suprir a demanda dos filhos, em detrimento da própria.

Palavras-chave: Cuidado pós-natal, Período pós-parto, Serviços de saúde.

\begin{abstract}
Objective: To identify possible gaps in the puerperal follow-up by the health service and the search for care by women in the first postpartum year. Methods: Prospective cohort, with 310 women, in a municipality in Paraná, between July 2013 and March 2015. A statistical analysis of the data was performed using the Chisquare test and the Spearman coefficient. Results: Statistical significance was found between being ill ( $p \leq$ 0.024); difficulty urinating ( $p \leq 0.011)$; self-perception of depressive illness ( $p \leq 0.007)$; performing actions to improve the signs and symptoms of depression ( $p \leq 0.007)$; oncotic cytology $(p \leq 0.000)$; looking for care up to 42 days after delivery $(p \leq 0.031)$ and between 43 days and one year after delivery $(p \leq 0.012)$; satisfaction with multidisciplinary care $(p \leq 0.001)$, physician $(p \leq 0.000)$, nursing $(p \leq 0.000)$; and the variables. The Spearman coefficient showed a very strong correlation between conducting an early home puerperal review (RPPD) and scheduling a late puerperal review at UBS at RPP $(r=0.953)$. Conclusion: The gap identified in the puerperium was one of care abandonment and the search for care evidenced the mothers' attempt to meet their children's demand, to the detriment of their own.
\end{abstract}

Keywords: Postnatal care, Postpartum period, Woman's health.

\footnotetext{
${ }^{1}$ Centro Estadual de Educação Tecnológica Paula Souza (CEETEPS), Barretos - SP.

*E-mail: aurea.quirino@etec.sp.gov.br

2 Universidade Estadual de Londrina (UEL), Londrina - PR.
} 


\section{RESUMEN}

Objetivo: Identificar posibles brechas en el seguimiento puerperal por parte del servicio de salud y la búsqueda de atención por parte de la mujer en el primer año posparto. Métodos: Cohorte prospectiva, con 310 mujeres, en un municipio de Paraná, entre julio de 2013 y marzo de 2015. Se realizó un análisis estadístico de los datos mediante la prueba de Chi-cuadrado y el coeficiente de Spearman. Resultados: Se encontró significación estadística entre estar enfermo ( $p \leq 0.024)$; dificultad para orinar $(p \leq 0.011)$; autopercepción de la enfermedad depresiva $(p \leq 0,007)$; realizar acciones para mejorar los signos y síntomas de la depresión ( $p \leq 0,007)$; citología oncótica $(p \leq 0,000)$; buscar atención hasta 42 días después del parto $(p \leq 0.031)$ y entre 43 días y un año después del parto $(p \leq 0.012)$; satisfacción con la atención multidisciplinaria $(p \leq 0,001)$, médico $(p \leq 0,000)$, enfermería $(p \leq 0,000)$; y las variables. El coeficiente de Spearman mostró una correlación muy fuerte entre la realización de una revisión puerperal domiciliaria temprana (RPPD) y la programación de una revisión puerperal tardía en UBS en RPP $(r=0,953)$. Conclusión: La brecha identificada en el puerperio fue de abandono del cuidado y la búsqueda del cuidado evidenció el intento de las madres por atender la demanda de sus hijos, en detrimento de la propia.

Palabras clave: Atención posnatal, Periodo posparto, Servicios de salud.

\section{INTRODUÇÃO}

O pós-parto é um período complexo do ciclo reprodutivo que envolve mudanças de ordem física, psicoemocional, comportamental, relacional e sociocultural, associadas a manifestações involutivas do organismo ao estado pré-gravídico (MAZZO MHSN, et al., 2018).

O primeiro ano após o parto é um momento de transição significante para as mulheres. Além das alterações fisiológicas, agregadas ao pós-parto, a mulher sofre mudanças psicossociais, como a transição para o papel materno, restabelecimento dos relacionamentos e, ainda, trabalha no intuito de satisfazer as necessidades do seu filho e de outras pessoas (FAHEY JO e SHENASSA E, 2013).

O cuidado deve fundamentar-se na história obstétrica, na assistência ao parto e nas reais necessidades, como a adaptação à nova realidade e a superação das dificuldades destas mulheres, recém-nascidos e familiares (TEIXEIRA EMB, et al., 2015).

Este vínculo pode ser garantido mediante a visita domiciliar (VD), considerada ferramenta fundamental para o cuidado prestado pelos profissionais do serviço de atenção primária em saúde no pós-parto (MINASI JM, et al., 2013; MIRMOLAEI ST, et al., 2014). Nesta oportunidade, a puérpera fica à vontade para expor suas dúvidas, sentimentos, queixas e questionamentos, enquanto o profissional vivencia suas reais condições de vida, podendo criar estratégias que potencializam os fatores de proteção da puérpera e dos demais (MINASI JM, et al., 2013).

Apesar de existirem políticas públicas de saúde voltadas para a saúde da mulher direcionadas ao ciclo gravídico-puerperal, é notório que a assistência ao período pós-parto se encontra em níveis inferiores quando comparada ao pré-natal e ao parto (BERNARDI MC, et al., 2011).

Souza ABQ e Fernandes BM (2014) encontraram um achado preocupante com relação à abordagem adotada pelos profissionais, ficando clara a valorização da saúde do bebê em detrimento da saúde da mulher. A falta de normatização da assistência ao período puerperal no âmbito da atenção primária é expressa pela busca por cuidado para o seguimento do recém-nascido (TEIXEIRA EMB, et al., 2015).

Neste sentido, é fundamental o entendimento do contexto em que as mulheres vivenciam o processo de procura por cuidado de saúde. A não procura do serviço para prevenção pode estar mais associada a barreiras reais, existentes entre a necessidade de cuidado e a oferta/acesso a este do que a valores pessoais determinantes de comportamentos (CARDELLI AAM e TANAKA ACA, 2012). É imprescindível que o profissional de saúde compreenda a integralidade da mulher, para que possa atuar como facilitador na assistência ao período pós-parto (BERNARDI MC, et al., 2011). 
Acredita-se que a mulher procura pouco o serviço de saúde para cuidar de si, e o seguimento, no período puerperal remoto, pela atenção primária não é eficaz. Diante do exposto, o objetivo do presente estudo foi identificar as possíveis lacunas no seguimento puerperal pelo serviço de saúde e a procura por cuidado pela mulher no primeiro ano pós-parto.

\section{MÉTODOS}

Trata-se de um estudo de coorte prospectivo com abordagem quantitativa, cuja população foi constituída por mulheres que realizaram o parto em uma maternidade de um município do Paraná. O estudo contemplou quatro etapas onde a primeira foi conduzida durante a internação hospitalar, envolvendo a identificação da mulher e consulta aos registros do prontuário sobre o parto e puerpério imediato; já a segunda etapa envolveu o retorno ambulatorial decorridos dez dias da alta hospitalar.

$\mathrm{Na}$ terceira etapa, procedeu-se a primeira VD, aos 42 dias pós-parto. E, finalmente, na quarta etapa, foi realizada a segunda VD, decorrido um ano após o parto. A coleta de dados aconteceu de julho de 2013 a março de 2015, sendo utilizada a técnica de entrevista estruturada para a coleta de dados, com o registro das respostas em um formulário semiestruturado.

A amostra inicial da coorte foi definida em 358 puérperas, sendo acrescidos $20 \%$ ( $N=429)$ para a primeira etapa. Os critérios de inclusão foram: residir na zona urbana, ser gestante de risco habitual ou intermediário e ser capaz de compreender e consentir a participação. Na segunda etapa todas as 429 participaram. A terceira etapa trabalhou com a amostra mínima $(\mathrm{N}=358)$ e na quarta etapa, a taxa de resposta foi de $86,6 \%$ $(\mathrm{N}=310)$. Os dados foram compilados no programa Microsoft Office Excel® 2007 e, posteriormente, transportados para o programa SPSS $\AA$, versão 20.0 .

$\mathrm{Na}$ análise univariada, foi empregado o Teste Qui-quadrado para busca de possíveis associações ( $\mathrm{p} \leq$ $0,05)$ e para verificar a existência de correlação no seguimento pelo serviço nas diferentes etapas da pesquisa, foi utilizado o Coeficiente de Spearman. O estudo foi aprovado pelo Comitê de Ética em Pesquisa Envolvendo Seres Humanos da Universidade Estadual de Londrina (CEP/UEL-PR) sob o número de parecer 234/2014, CAAE: 19352513.9.0000.5231 e seguiu as determinações da Resolução 466/12 do Conselho Nacional de Saúde.

\section{RESULTADOS}

As características sociais da população em estudo demonstraram mulheres jovens adultas $(88,3 \%)$ e adolescentes $(11,6 \%)$; com o companheiro (81,9\%); escolaridade de cinco a dez anos $(70 \%)$; renda familiar entre um e dois salários-mínimos (40,3\%); retornaram ao trabalho após quatro meses completos de licença maternidade $(45,2 \%)$ e em período inferior a quatro meses $(14,4 \%)$.

A procura por cuidado de saúde durante o primeiro ano após o parto (variável dependente) não se associou estatisticamente com as seguintes variáveis independentes: idade, situação conjugal, escolaridade, uso de tabaco, uso de bebida alcoólica, renda, retorno ao trabalho, mudanças físicas, autopercepção de doença depressiva, dificuldades na relação sexual devido ao parto normal, prática de exercícios físicos e doença.

Entretanto, é importante salientar que $96 \%$ das puérperas procuraram por cuidado de saúde e eram mulheres entre 20 e 30 anos (66\%), com companheiro (81,5\%), com escolaridade de cinco a dez anos (70\%), renda entre um e dois salários-mínimos $(47,1 \%)$ e a grande maioria (99\%) havia retornado ao trabalho, sendo que $60,2 \%$ com menos de quatro meses pós-parto.

Poucas eram as fumantes $(16,8 \%)$ e que faziam uso de bebida alcoólica $(23,6 \%)$. Pouco mais de $80 \%$ referiram ocorrência de mudanças físicas após o parto, autopercepção de doença depressiva $(83,5 \%)$, outras doenças $(60,9 \%)$ e práticas de atividades de lazer $(60,3 \%)$.

Houve associação estatisticamente significante entre a prática do autocuidado e a procura por cuidado na Unidade Básica de Saúde (UBS) no puerpério tardio $(p=0,031)$ e no remoto $(p=0,019)$. Percebe-se, também, uma tendência para associação estatística entre a prática do autocuidado e a procura por cuidado na maternidade dentro dos 30 dias pós-parto $(p=0,061)$. Entre as mulheres que praticavam o autocuidado $(63,2 \%) 89,3 \%$ não procuraram a maternidade dentro dos 30 dias após o parto (Tabela 1). 
Entre os motivos relatados, em relação à procura por cuidado na UBS até 42 dias pós-parto, observa-se que $76,8 \%$ foram para o atendimento do pediatra; $70,3 \%$ puericultura e somente $43,2 \%$ ginecologia. Já no puerpério remoto $95,8 \%$ procuraram por cuidado na UBS depois dos 43 dias pós-parto, sendo que os motivos foram: $93,6 \%$ pediatra, $96 \%$ puericultura e $52,9 \%$ ginecologia.

Tabela 1 - Distribuição das mulheres, segundo a procura por cuidado nas três etapas do estudo e a prática do autocuidado $(\mathrm{N}=310)$.

\begin{tabular}{|c|c|c|c|c|c|c|c|}
\hline \multirow{3}{*}{ Variáveis independentes } & \multicolumn{6}{|c|}{ Prática do autocuidado } & \multirow{3}{*}{ Valor de $p$} \\
\hline & \multicolumn{2}{|c|}{ Sim } & \multicolumn{2}{|c|}{ Não } & \multicolumn{2}{|c|}{ Total } & \\
\hline & $\mathbf{n}$ & $\%$ & $\mathbf{n}$ & $\%$ & $n$ & $\%$ & \\
\hline $2^{a}$ etapa & & & & & & & \\
\hline $\begin{array}{l}\text { Procura por cuidado na Maternidade dentro de } \\
30 \text { dias pós-parto }\end{array}$ & 196 & 100 & 114 & 100 & 310 & 100 & 196 \\
\hline Sim & 21 & 10,7 & 21 & 18,4 & 42 & 13,5 & 0 O61 \\
\hline Não & 175 & 89,3 & 93 & 81,6 & 268 & 86,5 & 0,061 \\
\hline $3^{\mathrm{a}}$ etapa & & & & & & & \\
\hline $\begin{array}{l}\text { Procura por cuidado na UBS até } 42 \text { dias pós- } \\
\text { parto }\end{array}$ & 196 & 100 & 114 & 100 & 310 & 100 & \\
\hline Sim & 187 & 95,4 & 101 & 88,6 & 196 & 63,2 & \\
\hline Não & 9 & 4,6 & 13 & 11,4 & 114 & 36,8 & $0,031^{*}$ \\
\hline $4^{\mathrm{a}}$ etapa & & & & & & & \\
\hline $\begin{array}{l}\text { Procura por cuidado na UBS depois de } 43 \text { dias } \\
\text { pós-parto }\end{array}$ & 195 & 100 & 115 & 100 & 310 & 100 & \\
\hline $\begin{array}{l}\text { Sim } \\
\text { Não }\end{array}$ & $\begin{array}{c}183 \\
12 \\
\end{array}$ & $\begin{array}{c}93,8 \\
6,2\end{array}$ & $\begin{array}{c}114 \\
1\end{array}$ & $\begin{array}{c}99,1 \\
0,9\end{array}$ & $\begin{array}{c}297 \\
13\end{array}$ & $\begin{array}{c}95,8 \\
4,2\end{array}$ & $0,019^{*}$ \\
\hline
\end{tabular}

Legenda: Teste Qui-quadrado $p \leq 0,05$.

Fonte: Silva AFAQ, et al., 2020.

A correlação entre as variáveis que caracterizaram o seguimento puerperal pelo serviço de saúde às mulheres nas quatro etapas em análise foi estatisticamente significativa. Observa-se que houve correlação muito forte entre realização de revisão puerperal precoce domiciliar (RPPD) e agendamento da revisão puerperal tardia na UBS na RPP $(r=0,953)$; correlação moderada entre VD de profissional de saúde entre 43 dias e 12 meses decorridos do parto e RPP $(r=0,415)$, agendamento da revisão puerperal tardia na UBS na RPP $(r=0,404)$ e agendamento da revisão puerperal remota $(r=0,461)$; fraca correlação entre agendamento da revisão puerperal remota e RPP $(r=0,245)$ e agendamento da revisão puerperal tardia na UBS na RPP ( $r$ $=0,257)$.

Tabela 2 - Correlação do seguimento puerperal pelo serviço de saúde nas quatro etapas em estudo ( $N=310)$.

\begin{tabular}{ccccccccc}
\hline & $\begin{array}{c}\text { Realização de } \\
\text { revisão puerperal } \\
\text { precoce domiciliar } \\
\text { (RPPD) }\end{array}$ & $\begin{array}{c}\text { Agendamento } \\
\text { da revisão } \\
\text { puerperal tardia } \\
\text { na UBS na } \\
\text { RPPD }\end{array}$ & $\begin{array}{c}\text { Agendamento } \\
\text { da revisão } \\
\text { puerperal } \\
\text { remota } \\
\text { (RPR) }\end{array}$ & $\begin{array}{c}\text { Visita domiciliar } \\
\text { de profissional de } \\
\text { saúde entre 43 } \\
\text { dias e 12 meses } \\
\text { decorridos do } \\
\text { parto }\end{array}$ \\
\cline { 2 - 9 } & $\mathbf{r}$ & $\mathbf{p}$ & $\mathbf{r}$ & $\mathbf{p}$ & $\mathbf{r}$ & $\mathbf{p}$ & $\mathbf{r}$ & $\mathbf{p}$ \\
\hline $\begin{array}{c}\text { Realização de revisão } \\
\text { puerperal precoce } \\
\text { domiciliar (RPPD) }\end{array}$ & - & - & 0,953 & $<0,0001$ & 0,245 & $<0,0001$ & 0,415 & $<0,0001$ \\
$\begin{array}{c}\text { Agendamento da revisão } \\
\text { puerperal tardia na UBS } \\
\text { na RPPD }\end{array}$ & 0,953 & $<0,0001$ & - & - & 0,257 & $<0,0001$ & 0,404 & $<0,0001$ \\
$\begin{array}{c}\text { Agendamento da revisão } \\
\text { puerperal remota (RPR) }\end{array}$ & 0,245 & $<0,0001$ & 0,257 & $<0,0001$ & - & - & 0,461 & $<0,0001$ \\
\hline $\begin{array}{c}\text { Visita domiciliar de } \\
\text { profissional de saúde } \\
\text { entre 43 dias e 12 meses } \\
\text { decorridos do parto }\end{array}$ & 0,415 & $<0,0001$ & 0,404 & $<0,0001$ & 0,461 & $<0,0001$ & - & - \\
\hline
\end{tabular}

Legenda: $r$ - Coeficiente de Spearman. Fonte: Silva AFAQ, et al., 2020. 
Em relação ao seguimento pelo serviço de saúde, é importante resgatar que a maioria das mulheres $(87,1 \%)$ realizou seis ou mais consultas pré-natais. No momento da alta hospitalar na maternidade, $77,4 \% \mathrm{e}$ $84,5 \%$ não receberam orientações sobre os sinais e sintomas de infecção puerperal e hemorragia pós-parto, respectivamente, e 16,1\% não foram contrarreferenciada a atenção primária. 52,3\% e 78\% não tiveram a RPPD e o agendamento da RPT na UBS, respectivamente, sendo que $62,9 \%$ receberam VD de um profissional de saúde entre 43 dias e 12 meses decorridos do parto.

Tabela 3 - Distribuição das puérperas, segundo o seguimento pelo serviço de saúde nas quatro etapas do estudo.

\begin{tabular}{|c|c|c|}
\hline Seguimento no pré-natal & $\mathbf{N}$ & $\%$ \\
\hline Número de consultas pré-natal & 310 & 100 \\
\hline 6 e mais & 270 & 87,1 \\
\hline 3 a 5 & 36 & 11,6 \\
\hline 0 a 2 & 4 & 1,3 \\
\hline Seguimento na alta hospitalar & $\mathbf{N}$ & $\%$ \\
\hline Orientações sobre infecção puerperal na alta hospitalar & 310 & 100 \\
\hline Sim & 70 & 22,6 \\
\hline Não & 240 & 77,4 \\
\hline Orientações sobre hemorragias na alta hospitalar & 310 & 100 \\
\hline Sim & 48 & 15,5 \\
\hline Não & 262 & 84,5 \\
\hline Contrarreferência à UBS orientada no momento da alta hospitalar & 310 & 100 \\
\hline Sim & 260 & 83,9 \\
\hline Não & 50 & 16,1 \\
\hline Seguimento do puerpério precoce ao tardio & $\mathbf{N}$ & $\%$ \\
\hline Revisão puerperal precoce domiciliar (RPPD) & 358 & 100 \\
\hline Sim & 171 & 47,7 \\
\hline Não & 187 & 52,3 \\
\hline Agendamento da revisão puerperal tardia na UBS & 358 & 100 \\
\hline Sim & 79 & 22 \\
\hline Não & 279 & 78 \\
\hline Seguimento no puerpério remoto & $\mathbf{N}$ & $\%$ \\
\hline $\begin{array}{l}\text { Visita domiciliar de profissional de saúde entre } 43 \text { dias e } 12 \text { meses } \\
\text { decorridos do parto }\end{array}$ & 310 & 100 \\
\hline $\operatorname{Sim}$ & 195 & 62,9 \\
\hline Não & 115 & 37,1 \\
\hline
\end{tabular}

Fonte: Silva AFAQ, et al., 2020.

Houve associação estatisticamente significativa entre VD de profissional de saúde entre 43 dias e 12 meses decorridos do parto (variável dependente) e relato de dificuldade para urinar $(p=0,011)$ e de doença no primeiro ano pós-parto $(p=0,024)$.

Entre as que referiram dificuldade para urinar (16,1\%) 9,6\% não receberam VD de algum profissional de saúde da atenção primária. Já entre as mulheres que relataram doença $53 \%$ também não tiveram este cuidado. As demais variáveis independentes não se associaram à dependente. A procura por cuidado para melhorar os sinais de sintomas preditivos de depressão foi relatada por $31,9 \%$ das mulheres e, dentre estas, $33,7 \%$ não receberam VD.

A prática do autocuidado não foi relatada por $37,1 \%$ das puérperas, sendo que dentre estas $36,5 \%$ também não receberam VD. Apenas $3,1 \%$ das mulheres que tiveram VD relataram como motivo desta, cuidados específicos às mesmas. No que diz respeito às orientações oferecidas durante a VD, $58,5 \%$ eram sobre cuidados com a criança; $23,6 \%$ com a mulher e a criança; $2 \%$ com a mulher e $11,8 \%$ não receberam nenhuma orientação. 
Tabela 4 - Relação entre a visita domiciliar do profissional de saúde entre 43 dias e 12 meses decorridos do parto e variáveis independentes em estudo $(\mathrm{N}=310)$.

\begin{tabular}{|c|c|c|c|c|c|c|c|}
\hline \multirow[t]{3}{*}{ Variáveis independentes } & \multicolumn{6}{|c|}{$\begin{array}{l}\text { Visita domiciliar de profissional de } \\
\text { saúde entre } \\
43 \text { dias e } 12 \text { meses decorridos do parto }\end{array}$} & \multirow{3}{*}{$\begin{array}{l}\text { Valor } \\
\text { de } p\end{array}$} \\
\hline & \multicolumn{2}{|c|}{ Sim } & \multicolumn{2}{|c|}{ Não } & \multicolumn{2}{|c|}{ Total } & \\
\hline & $\mathbf{n}$ & $\%$ & $\mathbf{n}$ & $\%$ & $\mathbf{n}$ & $\%$ & \\
\hline Tipo de parto & 195 & 100 & 115 & 100 & 310 & 100 & \\
\hline Normal espontâneo & 144 & 73,8 & 78 & 67,8 & 222 & 71,6 & \multirow{3}{*}{0,342} \\
\hline Normal instrumental & 4 & 2,1 & 5 & 4,4 & 09 & 2,9 & \\
\hline Cesárea & 47 & 24,1 & 32 & 27,8 & 79 & 25,5 & \\
\hline Laceração e/ou episiorrafia & 148 & 100 & 83 & 100 & 231 & 100 & \\
\hline Sim & 78 & 52,7 & 48 & 57,8 & 126 & 54,5 & \multirow{2}{*}{0,270} \\
\hline Não & 70 & 47,3 & 35 & 42,2 & 105 & 45,5 & \\
\hline $\begin{array}{c}\text { Dificuldades na relação sexual devido ao parto } \\
\text { normal }\end{array}$ & 144 & 100 & 80 & 100 & 224 & 100 & \\
\hline Sim & 13 & 9,0 & 6 & 7,5 & 19 & 8,5 & \multirow{2}{*}{0,451} \\
\hline Não & 131 & 91,0 & 74 & 92,5 & 205 & 91,5 & \\
\hline Dificuldade para urinar & 195 & 100 & 115 & 100 & 310 & 100 & \\
\hline Sim & 39 & 20,0 & 11 & 9,6 & 50 & 16,1 & \multirow{2}{*}{$0,011^{*}$} \\
\hline Não & 156 & 80,0 & 104 & 90,4 & 260 & 83,9 & \\
\hline Dificuldade para evacuar & 195 & 100 & 115 & 100 & 310 & 100 & \\
\hline Sim & 32 & 16,4 & 19 & 16,5 & 51 & 16,5 & \multirow{2}{*}{0,549} \\
\hline Não & 163 & 83,6 & 96 & 83,5 & 259 & 83,5 & \\
\hline Doença & 195 & 100 & 115 & 100 & 310 & 100 & \\
\hline Sim & 127 & 65,1 & 61 & 53,0 & 188 & 60,6 & \multirow{2}{*}{$0,024^{*}$} \\
\hline Não & 68 & 34,9 & 54 & 47,0 & 122 & 39,4 & \\
\hline Prática de autocuidado & 195 & 100 & 115 & 100 & 310 & 100 & \\
\hline Sempre & 122 & 62,6 & 73 & 63,5 & 195 & 62,9 & \multirow{2}{*}{0,485} \\
\hline Nunca & 73 & 37,4 & 42 & 36,5 & 115 & 37,1 & \\
\hline
\end{tabular}

Legenda: *Teste Qui-quadrado $p \leq 0,05$.

Fonte: Silva AFAQ, et al., 2020.

Observou-se associação estatisticamente significativa entre autopercepção de doença depressiva $(p=$ $0,007)$, procura por cuidado para melhorar sinais e sintomas preditivos de depressão $(p=0,007)$, citologia oncótica $(p=0,000)$, satisfação com o atendimento multiprofissional $(p=0,001)$, médico $(p=0,000)$ e de enfermagem $(p=0,000)$ e a variável dependente revisão puerperal remota (RPR).

Quanto ao acompanhamento por meio do programa de planejamento familiar, houve uma tendência para associação significativa entre o agendamento da RPR e realização deste $(p=0,056)$. As demais variáveis independentes não apresentaram associação estatística.

Entre as mulheres que não tiveram o agendamento da RPR, 80,1\% referiram autopercepção de doença depressiva; $37,6 \%$ não procuraram por cuidado para melhorar sinais e sintomas preditivos de depressão e $65 \%$ não realizaram citologia oncótica.

Em relação ao atendimento $77,8 \%$ das puérperas referiram satisfação com o cuidado multiprofissional e $89 \%$ tiveram agendamento da RPR; $52,5 \%$ ficaram insatisfeitas com o atendimento médico e entre estas $60,9 \%$ não tiveram o agendamento da RPR; $81,8 \%$ relataram satisfação com a enfermagem e $93 \%$ tiveram o agendamento da RPR. 
Tabela 5 - Relação entre agendamento da revisão puerperal remota na quarta etapa e variáveis independentes em estudo $(\mathrm{N}=310)$.

\begin{tabular}{|c|c|c|c|c|c|c|c|}
\hline \multirow{3}{*}{$\begin{array}{c}\text { Variáveis } \\
\text { independentes }\end{array}$} & \multicolumn{6}{|c|}{ Agendamento da revisão puerperal remota } & \multirow{3}{*}{$\begin{array}{l}\text { Valor } \\
\text { de } p\end{array}$} \\
\hline & \multicolumn{2}{|c|}{ Sim } & \multicolumn{2}{|c|}{ Não } & \multicolumn{2}{|c|}{ Total } & \\
\hline & $\mathbf{n}$ & $\%$ & $\mathbf{n}$ & $\%$ & $\mathbf{n}$ & $\%$ & \\
\hline $\begin{array}{l}\text { Autopercepção de } \\
\text { doença depressiva }\end{array}$ & 104 & 100 & 206 & 100 & 310 & 100 & $0,007^{*}$ \\
\hline Sim & 95 & 91,3 & 165 & 80,1 & 260 & 83,9 & \\
\hline Não & 9 & 8,7 & 41 & 19,9 & 50 & 16,1 & \\
\hline $\begin{array}{l}\text { Realização de ações para } \\
\text { melhorar os sinais e } \\
\text { sintomas de depressão }\end{array}$ & 95 & 100 & 165 & 100 & 260 & 100 & $0,007^{*}$ \\
\hline Sim & 74 & 77,9 & 103 & 62,4 & 177 & 68,1 & \\
\hline Não & 21 & 22,1 & 62 & 37,6 & 83 & 31,9 & \\
\hline Método contraceptivo & 104 & 100 & 206 & 100 & 310 & 100 & 0,391 \\
\hline Sim & 89 & 85,6 & 180 & 87,4 & 269 & 86,8 & \\
\hline Não & 15 & 14,4 & 26 & 12,6 & 41 & 13,2 & \\
\hline Citologia oncótica & 104 & 100 & 206 & 100 & 310 & 100 & $0,000^{*}$ \\
\hline Sim & 66 & 63,5 & 72 & 35,0 & 138 & 44,5 & \\
\hline Não & 38 & 36,5 & 134 & 65,0 & 172 & 55,5 & \\
\hline $\begin{array}{l}\text { Satisfação com o } \\
\text { atendimento } \\
\text { multiprofissional }\end{array}$ & 100 & 100 & 197 & 100 & 297 & 100 & $0,001^{*}$ \\
\hline Satisfeita & 89 & 89,0 & 142 & 72,1 & 231 & 77,8 & \\
\hline Insatisfeita & 11 & 11,0 & 55 & 27,9 & 66 & 22,2 & \\
\hline $\begin{array}{l}\text { Satisfação com o } \\
\text { atendimento médico }\end{array}$ & 100 & 100 & 197 & 100 & 297 & 100 & $0,000^{*}$ \\
\hline Satisfeita & 64 & 64,0 & 77 & 39,1 & 141 & 47,5 & \\
\hline Insatisfeita & 36 & 36,0 & 120 & 60,9 & 156 & 52,5 & \\
\hline $\begin{array}{l}\text { Satisfação com o } \\
\text { atendimento de } \\
\text { enfermagem }\end{array}$ & 100 & 100 & 197 & 100 & 297 & 100 & $0,000^{*}$ \\
\hline Satisfeita & 93 & 93,0 & 150 & 76,1 & 243 & 81,8 & \\
\hline Insatisfeita & 7 & 7,0 & 47 & 23,9 & 54 & 18,2 & \\
\hline
\end{tabular}

Legenda: *Teste Qui-quadrado $p \leq 0,05$.

Fonte: Silva AFAQ, et al., 2020.

\section{DISCUSSÃO}

O pré-natal é um exemplo concreto de ação programática que aumenta o vínculo, a conscientização e, com isso, a procura por cuidado, sendo este refletido até mesmo no puerpério (BRASIL, 2013). É considera elevada a taxa superior a $80 \%$ de cobertura de sete ou mais consultas de pré-natal (LONDRINA, 2013). $O$ estudo de Regassa N (2011), revelou que o nível de cuidados pré-natais e cuidados pós-natais foi de $77,4 \%$ e $37,2 \%$, respectivamente. Percebe-se que no pré-natal a utilização de serviços foi geralmente boa, enquanto no cuidado pós-natal foi muito baixa em comparação a outros grupos da população da região.

No que tange às orientações oferecidas na maternidade foram realizadas quanto à contrarreferência, mas houve pouca ênfase nas orientações sobre a infecção puerperal e as hemorragias na alta hospitalar neste estudo. Na alta hospitalar, a falha nas orientações e a ausência de responsabilidade das instituições de saúde com o paciente que recebeu alta e foi para a comunidade pode contribuir para que as mulheres realizem medidas prejudiciais à sua saúde no âmbito domiciliar (ACOSTA DF, et al., 2012; SILVA LR, et al., 2012).

No estudo de Santos FAPS, et al. (2013), os autores falam de implicações na prática assistencial à mulher no período pós-parto, sendo importante que a puérpera antes de sair da maternidade saiba a que o tipo de apoio pode recorrer. Estes elementos são cruciais para a prática de enfermagem mais interventiva numa abordagem sistêmica de suporte técnico e relacional junto à puérpera e de seus familiares. A ausência de 
alinhamento no âmbito da estrutura e dos fluxos de funcionamento do sistema e/ou serviço de saúde pode impactar de maneira negativa no relacionamento entre o profissional e o usuário (VIEGAS APB, et al., 2015).

A infecção puerperal e a hemorragia pós-parto são as intercorrências mais comuns no pós-parto. No estudo de Teixeira EMB, et al. (2015), na avaliação da tríade abdômen, incisão cirúrgica ou períneo e lóquios foram identificados índices altos e alarmantes entre os cuidados que não foram ofertados tanto na revisão puerperal precoce ambulatorial quanto na domiciliar, do mesmo modo que as orientações de autocuidado para prevenção e identificação destas possíveis complicações.

Evidencia-se que o baixo conhecimento da puérpera frente aos sinais e sintomas de infecção e hemorragia, assim como a ausência de esclarecimentos sobre os cuidados específicos desse período, aumenta o espaço para condutas baseadas em crenças e atitudes negativas. No Brasil e no município em estudo, a atuação dos profissionais para o seguimento puerperal é direcionada para a realização de revisões puerperais programáticas, em dois momentos: precoce (entre sete e dez dias pós-parto) e tardia (até 42 dias de pós-parto). Não existe a proposição e acompanhamento programático após os 43 dias do parto, estendendo este período até um ano. Beleza ACS, et al. (2012), sugerem a realização de estudos nacionais que analisem o acompanhamento da puérpera no período remoto.

Os três períodos puerperais acompanhados neste estudo tiveram seguimento, entretanto, voltado às orientações e cuidados para os filhos das puérperas. Esta situação foi evidenciada por Teixeira EMB, et al. (2015), em relação a primeira, segunda e terceira etapas deste estudo e manteve-se na quarta etapa. Esta lacuna no atendimento às necessidades da mulher, principalmente no puerpério remoto, é gritante e continua a ser desvalorizada pelas políticas de saúde específicas para este grupo, caracterizando o abandono da mulher em um período pleno de fatores que podem determinar riscos à sua saúde. Em seu estudo Corrêa MSM, et al. (2017), observou que no atendimento as ações são voltadas para a à saúde da criança, com isso as mulheres não são devidamente contempladas em suas necessidades.

Exemplo desta lacuna é o Protocolo da Atenção Básica: Saúde das Mulheres que apresenta apenas um parágrafo em suas proposições referente ao puerpério remoto (BRASIL, 2015). Da mesma forma, as ações estabelecidas pela Rede Mãe Paranaense, enfatizam a captação precoce da gestante; acompanhamento prénatal; estratificação de risco das gestantes e das crianças; hospital de referência; VD precoce para puérperas e recém-nascidos até o quinto dia pós-parto, agendamento dos seguimentos apropriados à mulher e à criança na UBS, sem, entretanto, especificar a realização da VD por um profissional de saúde no puerpério remoto (PARANÁ, 2013).

Neste sentido, a pouca ênfase dada a este período influencia a prática do profissional de saúde, que prioriza o seguimento puerperal precoce e tardio em detrimento do remoto e, quando o faz, direciona seus cuidados à criança. Neste estudo esta situação foi evidente, além do fato de que as VD no puerpério remoto foram decorrentes de problemas de saúde e não tiveram caráter preventivo ou de promoção desta. Existe o risco de morbimortalidade nestas mulheres no período pós-parto, sendo a atenção primária em saúde primordial no oferecimento do cuidado necessário, além da responsabilidade na resolução dos impasses de saúde, revelando a necessidade do aprimoramento de ações efetivas e eficazes no intuito de atender as exigências de saúde das mulheres no puerpério (BARATIERI T e NATAL S, 2019).

Vale ressaltar que no presente estudo, por meio da correlação do acompanhamento pelo serviço de saúde nas quatro etapas, identificou-se que revisão puerperal precoce domiciliar foi eficaz para o retorno da mulher à UBS para efetivar a revisão puerperal tardia, bem como para o acompanhamento durante o primeiro ano após o parto. A revisão pós-parto é um meio de diagnóstico precoce de patologias e, com isso, de complicações. Acredita-se que deve levar em consideração as características e particularidades de cada puérpera. Estas ações podem ser desenvolvidas tanto no âmbito domiciliar quanto nos serviços de saúde (SANTOS FAPS, et al., 2013). A VD realizada por profissionais de saúde pode influenciar de maneira positiva e eficaz na melhora de comportamentos saudáveis pelas mulheres nos países menos desenvolvidos (MIRMOLAEI ST, et al., 2014). Recentemente, o estudo Nascer no Brasil promoveu o acompanhamento de grupo de mulheres até 12 meses de pós-parto, para avaliar desfechos maternos e neonatais (GAMA SGN, et al., 2014). 
Fato também relevante no presente estudo foi o não agendamento da revisão puerperal remota (RPR), que distanciou a mulher do programa de planejamento familiar. No estudo de Abera Y, et al. (2015), os autores identificaram que informar sobre o planejamento familiar no pós-parto é fundamental para uma melhora na sobrevivência materna, neonatal e infantil, pois permite que as mulheres possam alcançar um intervalo interpartal saudável e seguro.

O comportamento das mulheres em relação aos métodos contraceptivos no período pós-parto geralmente é diferente das outras vezes no ciclo de vida devido aos papéis e à presença de alterações emocionais adicionais ao período vivenciado. Corroborando com o presente estudo, Souza ABQ e Fernandes BM (2014), que avaliaram a implantação de uma diretriz voltada ao puerpério na atenção primária no Brasil, ao realizar uma avaliação sobre o que existe relacionado a recomendações para este período, não encontrou propostas pertinentes ao puerpério remoto.

Em relação à procura por cuidado de saúde, as mulheres eram jovens, com situação social estável e prática de atitudes saudáveis, características de mulheres que cuidavam de seu bem-estar. Portanto, podese inferir que esta procura pelo serviço de saúde teve dois fatores: o primeiro possivelmente foi uma resposta às ações programáticas ofertadas na atenção primária para este grupo; e o segundo foi decorrente de intercorrências autopercebidas. A prática do autocuidado favorece a não presença de intercorrências, reduzindo a utilização desnecessária do serviço. Da mesma forma, a prática do autocuidado relaciona-se com a consciência sobre a importância da procura por cuidado de saúde, por meio de atitudes positivas em prevenção (CARDELLI AAM e TANAKA ACA, 2012).

Outro aspecto evidente foi a baixa escolaridade como um fator negativo para a procura por cuidado de saúde. Um dos objetivos de Desenvolvimento do Milênio das Nações Unidas é a educação, reconhecida como meio para a consolidação dos direitos e edificação da autonomia individual e coletiva (BRASIL, 2013).

A procura por cuidado de saúde nas quatro etapas de seguimento das mulheres evidenciou que a prática do autocuidado se concretizou quando a mulher não procurou a maternidade municipal, fato coerente com a situação de mulheres que tiveram ações preventivas logo após seus partos, evitando problemas de saúde associados a estes. Da mesma forma, a prática do autocuidado também se concretizou quando estas mulheres procuraram pelo serviço de saúde, tanto no puerpério tardio como no remoto. Segundo Oliveira JCS, et al. (2015), revinda da mulher ao serviço de saúde precisa ser ressaltada no decorrer do pré-natal e na sua permanência na maternidade.

Os sinais preditivos de depressão, comuns no período puerperal, ocorreram entre as mulheres deste estudo e não foram determinantes para a procura por cuidado de saúde. Frota CA, et al. (2020), em seu estudo constatou a depressão pós-parto, sendo o transtorno mais referido, tendo potencial para causar graves consequências à saúde da mulher.

Na pesquisa Nascer no Brasil, 26\% das mães entre seis e 18 meses de pós-parto apresentavam depressão, sendo que esta patologia pode influenciar negativamente a saúde da mulher, afetando sua relação com a família e com o bebê (LEAL MC, et al., 2014).

Apesar do seguimento puerperal remoto não ter sido voltado às necessidades das mulheres, estas ficaram satisfeitas com o atendimento multiprofissional e de enfermagem, mas a maioria classificou como insatisfeito o atendimento médico da UBS. A satisfação dos usuários está associada à qualidade do serviço. Com o objetivo de aprimorar os serviços de saúde, é necessário apurar a satisfação daqueles que utilizam a atenção primária em saúde (MORAES VD, et al., 2014).

Em seu estudo Cardelli AAM e Tanaka ACA (2012), apreenderam nas falas das mulheres que as crenças sobre as dificuldades de acesso à saúde aparecem relacionadas a diferentes fatores, como: insatisfação com a organização e tipo de atendimento do serviço; adversidades no acesso geográfico; falta de privacidade e de opção pelo profissional que presta o atendimento; diferenciação social existente no serviço público e privado; inadequação do serviço público às necessidades da demanda e dificuldade pessoal em procurar por cuidado. A desarticulação do serviço e o relacionamento fragilizado com a puérpera podem ser refletidos em insatisfação com o atendimento recebido (TEIXEIRA EMB, et al., 2015). 


\section{CONCLUSÃO}

Concluiu-se, apesar da limitação do estudo, determinada pela opção de unidade de análise que foi a perspectiva da puérpera que houve seguimento das mulheres na atenção primária, principalmente das ações programáticas no pré-natal, alta hospitalar e nos períodos puerperais precoce e tardio. O puerpério remoto foi caracterizado pelo abandono assistencial, sendo que, quando o cuidado ocorreu, foi direcionado à criança. $\mathrm{A}$ procura por cuidado evidenciou a tentativa das mães de suprir a demanda de seus filhos, em detrimento das suas próprias necessidades. O seguimento puerperal e a procura por cuidado quando desempenhados de forma efetiva pode beneficiar no reconhecimento de possíveis complicações pertinentes deste período, além disso contribuir para o bem-estar biológico, físico e social do binômio e da família.

\section{REFERÊNCIAS}

1. ABERA Y, et al. Postpartum contraceptive use in Gondar town, Northwest Ethiopia: a community based crosssectional study. Women's Health. 2015; (15) 19: 2-8.

2. ACOSTA DF, et al. Influências, crenças e práticas no autocuidado das puérperas. Revista da Escola de Enfermagem - USP. 2012; 46 (6): 1327-33.

3. BARATIERI T, NATAL S. Ações do programa de puerpério na atenção primária: uma revisão integrativa. Ciênc. saúde coletiva. 2019; 24(11): 4227-4238.

4. BELEZA ACS, et al. Mensuração e caracterização da dor após episiotomia e sua relação com a limitação de atividades. Revista Brasileira de Enfermagem. 2012; 65 (2): 264-68.

5. BERNARDI MC, et al. Visita domiciliária puerperal como estratégia de cuidado de enfermagem na atenção básica: revisão integrativa. Revista da Rede de Enfermagem do Nordeste. 2011; 12 (esp.):1074-80.

6. BRASIL. 2015. In: Ministério da Saúde, Protocolos da Atenção Básica: saúde das mulheres. Instituto Sírio-Libanês de Ensino e Pesquisa, Brasília, DF, 2015. 188 p.

7. BRASIL. 2013. In: Ministério da Saúde, Secretaria de Políticas para as Mulheres. Plano Nacional de Políticas para as Mulheres. Brasília, DF, 2013. 114 p.

8. CARDELLI AAM, TANAKA ACA. O papel das crenças e percepções de mulheres na vivência do processo saúdedoença. Ciência, Cuidado e Saúde. 2012; 11 (supl.): 108-14.

9. CORREA MSM, et al. Acolhimento no cuidado à saúde da mulher no puerpério. Cad. Saúde Pública. 2017; 33(3): e00136215.

10. FAHEY JO, SHENASSA E. Understanding and meeting the needs of women in the postpartum period: the perinatal maternal health promotion model. Journal of Midwifery \& Women's Health. 2013; 58 (6): 613-21.

11. FROTA CA, et al. A transição emocional materna no período puerperal associada aos transtornos psicológicos como a depressão pós-parto. Revista Eletrônica Acervo Saúde. 2020; 48 : e3237.

12. GAMA SGN, et al. Fatores associados à cesariana entre primíparas adolescentes no Brasil, 2011-2012. Cadernos de Saúde Pública. 2014; 30 (1): 117-27.

13. LEAL MC, et al. Sumário executivo temático da pesquisa nascer no Brasil. Cadernos de Saúde Pública. $2014 ; 30$ (Suppl.1): 43-7.

14. LONDRINA. 2013. In: Secretaria Municipal de Saúde, Londrina. Paraná. Plano Municipal de Saúde 2014-2017. 2013. $221 \mathrm{p}$.

15. MAZZO MHSN, et al. Percepção das puérperas sobre seu período pós-parto. Investigación en Enfermería: Imagen y Desarrollo. 2018; 20(2): 2027-128X.

16. MINASI JM, et al. Perfil obstétrico e intercorrências de puérperas assistidas em visita domiciliária. Revista da Rede de Enfermagem do Nordeste. 2013; 14 (4): 757-64.

17. MIRMOLAEI ST, et al. Comparison of effects of home visits and routine postpartum care on the healthy behaviors of Iranian low-risk mothers. International Journal of Preventive Medicine. 2014; 5 (1): 61-8.

18. MORAES VD, et al. Estudo sobre dimensões da avaliação da Estratégia Saúde da Família pela perspectiva do usuário. Physis: Revista de Saúde Coletiva. 2014; 24 (1): 127-46.

19. OLIVEIRA JCS, et al. Assistência ao pré-natal realizada por enfermeiros: o olhar da puérpera. R. Enferm. Cent. O. Min. 2015; 5(2):1613-1628.

20. PARANÁ. Secretaria de Estado da Saúde do Paraná. Superintendência de Atenção à Saúde. Linha Guia da Rede Mãe Paranaense, 2013.

21. SANTOS FAPS, et al. Puerpério e revisão pós-parto: significados atribuídos pela puérpera. Revista Mineira de Enfermagem. 2013; 17 (4): 854-858.

22. SILVA LR, et al. Enfermagem no puerpério: detectando o conhecimento das puérperas para o autocuidado e cuidado com o recém-nascido. Revista de Pesquisa: Cuidado é Fundamental. 2012; 4 (2): 2327-37.

23. SOUZA ABQ, FERNANDES BM. Diretriz para assistência de enfermagem: ferramenta eficaz para a promoção da saúde no puerpério. Revista da Rede de Enfermagem do Nordeste. 2014; 15 (4): 594-604.

24. TEIXEIRA EMB, et al. Early postpartum review: analysis of the programmatic actions offered in primary health care. Business and Management Review - Special Health Issue Brazil. 2015; (11): 82-92.

25. VIEGAS APB, et al. Fatores que influenciam o acesso aos serviços de saúde na visão de profissionais e usuários de uma Unidade Básica de referência. Saúde Sociedade. 2015; 24 (1): 100-12. 